\title{
An Ecological Comparison Study On The Causal Association Between Leptospirosis and Moyamoya Disease in Hubei, China, 2017-2019
}

\section{Ma Zhiyang}

Zhongnan Hospital of Wuhan University

\section{Xiong Wang}

China University of Geosciences (Wuhan)

\section{Meifang Li}

Jiangxi Normal University

\section{Da Zhou}

Information Statistical Bureau, Hubei Health Committee

Jianjian Zhang

Zhongnan Hospital of Wuhan University

\section{Lu Ma}

Wuhan University

\section{Xiang Li}

Zhongnan Hospital of Wuhan University

\section{Wei Wei}

Zhongnan Hospital of Wuhan University

\section{Xiaohui Wu}

Zhongnan Hospital of Wuhan University

\section{Xiangyan Chen}

Department of Health Technology and Informatics, the Hong Kong Polytechnic University

\section{Keyao Zhou}

Zhongnan Hospital of Wuhan University

\section{Zenghui Huang}

Wuhan University

Jincao Chen ( $\square$ chenjincao2012@163.com )

Zhongnan Hospital of Wuhan University

\section{Research Article}

Keywords: Moyamoya disease, Ecological study, Spatial epidemiology, Leptospirosis, Environmental risk factors 
Posted Date: July 15th, 2021

DOl: https://doi.org/10.21203/rs.3.rs-708790/v1

License: (c) (i) This work is licensed under a Creative Commons Attribution 4.0 International License. Read Full License 


\section{Abstract}

\section{Background and Purpose}

The importance of environmental factors (especially leptospirosis) in moyamoya disease (MMD) has not been clarified. Here we investigated the epidemiological characteristics of MMD under perspective of ecology in Hubei province, China.

\section{Methods}

We conducted a population-based study to describe the epidemiologic characteristics of MMD in Hubei province between 2017 and 2019. The regional clusters of the hot spots (high incidence) and cold spots (low incidence) of MMD were identified using the spatial statistical method. To evaluate the role of leptospirosis in MMD, we performed an ecological comparison study to evaluate whether the socioeconomic and environmental variables of hot spots are more suitable for leptospirosis spread.

\section{Results}

The average annual age-adjusted incidence of MMD was 2.85 per 100000 person-years from 2017 to 2019. The middle-aged had higher incidence of MMD than the children. There existed an obvious geographic distribution of MMD at county level that the average annual age-adjusted incidence of hot spots was about 8 times than the cold spots. The hot spots were identified mainly in the low mountainous and hilly terrain, while cold spots were located in the Jianghan Plains. Compared to cold spots, the hot spots had larger cattle density ( 28.9 vs $7.7, P=0.002)$, higher percentages of rice field ( $80.3 \%$ vs $35.7 \%, P=0.002$ ), and lower elevation (33.6 vs $157.4, P=0.001$ )

\section{Conclusions}

We identified the obvious geographic distribution of MMD in the province, which initially strengthened the importance of environmental factors of this disease. Moreover, we preliminarily identified that people who lived in the low elevation regions with close contact to the cattle and rice field has a high risk of MMD. Future studies are needed to explore the potential environmental factors in MMD, especially for early-life exposure to leptospirosis.

\section{Introduction}

Moyamoya disease (MMD) is a cerebrovascular disorder with unknown pathogenesis, characterized by chronic progressive occlusion of the terminal internal carotid artery and dilated collateral microvessels near the base skull, developing collateral circulation like "puff of smoke" on angiography ${ }^{1,2}$. Although MMD is not a common disease, it is the major reason for pediatric and young adult stroke in East Asian regions ${ }^{3}$. However, the etiology of MMD remain largely unknown. 
Genetical epidemiology has demonstrated that the ring finger protein 213 (RNF213) is a major susceptible gene for MMD among the East Asian populations ${ }^{4,5}$. However, the RNF213 mutation cannot explain the etiology of MMD alone because of its quite low penetrance in normal population ${ }^{6}$. Thus, except for genetic factors, some environmental factors might trigger the start of MMD. In the 20th century when leptospirosis was epidemic in China, researchers reported that leptospiral cerebral vasculitis (LCV), one of the late complications of leptospirosis, which showed similar clinical, pathological as well as angiographic features with $\mathrm{MMD}^{7,8}$. However, the association between leptospirosis and MMD has not been clarified.

Leptospirosis is a globally important zoonotic disease which are transmitted to humans directly by contact with infected urine shed by carrier animals (wild or domestic) or indirectly via leptospiralcontaminated soil, water or food, especially in times of flooding. A lot of factors are determinants of the spread of the disease, including socioeconomic status, particular environmental conditions, and behavioral and occupational habits of humans ${ }^{9}$. In the 20th century in some rural regions of China, both the old life habits of widely free-ranged domestic animal (especially pig and cattle) and collectively manual labor in rice field created best conditions for leptospirosis spread, which could lead to large leptospirosis outbreak during extremely heavy rainfall or flood disasters ${ }^{10,11}$. Due to the leptospirosis is an acute infectious disease while MMD is a chronic cerebrovascular disease, which means that there would exist a latent period from leptospirosis to MMD if the two diseases did have a causal association. Thus, considering the particular environmental conditions for leptospirosis might minimally change with time, we investigate the association between leptospirosis and MMD from the perspective of ecology.

The ecological comparison study (ECS) could provide essential clues for detecting putative environmental causes of diseases ${ }^{12}$. After demonstrating higher and lower incidence clusters of a disease, we can identify the potentially responsible exposures for such clusters. In this study, our primary objective is to identify the regional clusters of MMD in Hubei, China, and then we applied the ECS to evaluate whether the socioeconomic and environmental variables of high clusters of MMD are more suitable for leptospirosis outbreak.

\section{Methods}

\subsection{Study area}

This study focused on Hubei $\left(29^{\square} 05^{\prime}-33^{\square} 20^{\prime} \mathrm{N}, 108^{\square} 21^{\prime}-116^{\square} 07^{\prime} \mathrm{E}\right)$, a province of the Central China, has an area of 185900 square kilometers and a population of 59.2 million at the end of 2018. Chinese Han consists $96 \%$ of all the population according to the sixth population overall survey (2010) of Hubei province. The province includes 16 cities and one forest district (Shennongjia), and the 16 cities are further divided into 38 counties, 25 prefecture-level cities, and 39 districts (Figure 1A). For brevity, the county described in this study also includes the district and prefecture-level city. This province is located between the Yangtze River and the Han River, and it is dominated by plains in the south-central, with surrounding mountains in the west and hills in the east (Figure 1B). Most areas have a subtropical 
monsoon humid climate, with an average annual temperature of $15-17^{\circ} \mathrm{C}$ and average annual precipitation of $800-1600 \mathrm{~mm}$. Due to the diverse geographic characteristics, Hubei is one of the provinces that were mostly affected by flood disasters and leptospirosis. More importantly, it was the Hubei researchers who firstly identified that MMD could resulted from leptospirosis. Thus, it is appropriate to select this province for studying the association of leptospirosis and MMD.

\subsection{Data source}

We collected and collated the available data of patients with MMD from the inpatient database of Hubei Health Committee (HHC) from October 2016 to July 2020. Since 2016, HHC started to collect inpatient information from all tertiary and secondary hospitals of the Hubei province. In China, tertiary and secondary hospitals generally represent regional medical centers at the city and county levels, respectively, which can mostly perform the cerebrovascular examination for MMD diagnosis. For each MMD patient in the database, the retrieved information included demographic characteristics (age, sex), home address, admission date, discharge diagnosis, and corresponding International Classification of Diseases (ICD-10) codes. The patients were excluded if they were repeated hospitalization or the addresses of whom were out of Hubei province or the key information (age, sex, address) was absent or diagnosed in 2016 and 2020.

The demographic information was acquired from the Statistical Yearbook of Hubei province in relevant years (2017-2019). Data from the sixth population overall survey (2010) of Hubei province served as a standard population to calculate the sex- or age-adjusted incidence rate of MMD. Geographical data of both city and county boundaries were accessed from digital maps from the National Fundamental Geographic Information System, China (2014). The digital elevation model (DEM) used to reflect the landform was presented by the author in this study.

Data on socioeconomic and environmental variables at county level were acquired from the Statistical Yearbook of Hubei province (2018).

\subsection{Demographic features analysis}

For demographic features analysis, we described the median age onset and the sex ratio. From 2017 to 2019, we conducted a population-based study on the AAIR at province level. The AAIR was calculated by the number of MMD cases divided by the resident population at the end of the year and was adjusted by the standard population in 2010. We adjusted the crude incidence by age for considering a population bias between counties.

\subsection{Regional distribution and Hot-spot analysis}

The software ArcGIS (10.5 ESRI, Redlands, CA, USA) was used to illustrate the heterogeneity of the geographic distribution of MMD. We calculated the annual AAIR of 103 counties from 2017 to 2019, and the results were displayed with different colors on the map. To examine the hot and cold spots of MMD, 
we applied the spatial statistical method of Getis-Ord Gi*17. Hot spot is defined by a county that is significantly clustered with a high AAIR of MMD, and cold spot is defined by a county that is significantly clustered with a low AAIR of the disease. Not cluster means not statistically significant.

In this study, we performed the hot-spot analysis at each year from 2017 to 2019 . Thus, each county would have likely to be identified as three different combinations, including hot spot, cold spot, or not cluster. To solve the problem that a county might be identified with different results, we set the following regulations to define a more accurate hot and cold spot. If a county is identified as hot spot for at least two times, we define this county as a real hot spot. Similarly, if a county is identified as cold-spot for at least two times, we define it as a real cold spot. If a county is identified as hot and cold spots coexisted or other possible combinations, we define it as not cluster.

\subsection{Differences between hot and cold spots}

After identifying the hot and cold spots, we compared the potential risk variables of leptospirosis between hot and cold spots. Socioeconomic status was evaluated by urbanization rate, per capita gross domestic product (GDP, 1 000\$), per capita disposable income of urban residents (DIOUR, $1000 \$$ ), disposable income of rural residents (DIORR, $1000 \$$ ), and the sanitary conditions. Hospital beds and medical workers (mainly doctors and nurses) per 1000 people were selected to represent sanitary conditions. Particular natural environments are supposed to increase the risk of leptospirosis outbreak, including the elevation, the domestic animal density, and the proportion of rice field. The low elevation regions that are easier to be affected by flood disasters, where individuals are supposed to suffer higher risk of leptospirosis outbreak. Domestic animal density, including pig and cattle which were essential infectious hosts of leptospirosis in China, defined as the number of animals divided by the county area. The proportion of rice field in cultivated land indicates the risk of contact with Leptospira-contaminated water in a county.

\subsection{Statistical analysis}

Statistical analyses were performed using SPSS statistical software (version 23.0, IBM Corp, Armonk, NY, 2014). $P$ values were calculated using 2 -tailed tests, and $P<0.05$ was considered statistically significant. From 2017 to 2019, to evaluate the province level incidence, we adjusted the crude incidence by the standard population at the age in bands of 5 years in 2010. All the categorical variables were shown as absolute numbers with percentages. The annual AAIR and socioeconomic and environmental variables in hot and cold spots were shown as the median (IQR) and were compared using the Wilcoxon rank-sum test.

\section{Results}

\subsection{Basic epidemiological characteristics}


Totally, we collected 10498 cases of MMD between October 2016 and July 2020. of which 3434, 274, $354,9,304$, and 694 patients were excluded for repeated hospitalization, unclear address, out of the province, age absent, diagnosed in 2016, and 2020, respectively. Finally, we included 5429 patients in this study from 2017 to 2019 . The juvenile patients accounted for only $1 \%(55 / 5429)$. The median age at admission was 52 (IQR, 46-60) years old, which showed a steadily increasing trend from 2017 to 2019. Both women and men followed a similar age distribution pattern, without a female predominance (Table 1 and Fig. 2). From 2017 to 2019, the province-level AAIR was 2.11-3.38 per 100000 personyears, with an average at 2.85 (Table 1). The annual incidence in different age groups exhibited two huge peaks that the first peak located at 50-54 years and the second one at 65-69 years. In addition, we observed one smaller peak aged at 25-34 years, with a sharp increase since the age of around 40 (Fig. 2).

Table 1

The basic characteristics of MMD in Hubei province from 2017 to 2019

\begin{tabular}{|lllll|}
\hline Characteristics & $\mathbf{2 0 1 7}$ & $\mathbf{2 0 1 8}$ & $\mathbf{2 0 1 9}$ & Total \\
\hline Resident population (million) & 58.85 & 59.02 & 59.17 & $59.01^{\dagger}$ \\
\hline Number of patients & 1388 & 1891 & 2150 & 5429 \\
\hline Age $<18$, no. (\%) & $22(1 \cdot 6)$ & $12(0 \cdot 6)$ & $21(1 \cdot 0)$ & $55(1.0)$ \\
\hline Age, median (IQR) & $51(44-59)$ & $52(46-61)$ & $53(46-61)$ & $52(46-60)$ \\
\hline $\begin{array}{l}\text { Sex ratio (women/men) } \\
\text { AAIR, per 100 000 person- }\end{array}$ & 1.0 & 1.0 & 1.1 & 1.0 \\
years & $(695 / 693)$ & $(950 / 941)$ & $(1109 / 1041)$ & $(2754 / 2675)$ \\
\hline
\end{tabular}

Note: AAIR, age adjusted incidence rate. ${ }^{\dagger}$ The average population from 2017 to 2019; *Average annual AAIR from 2017 to 2019.

\subsection{Regional Distribution}

From the disease map, it was apparent that higher MMD incidence rates were observed in the southeastern and north-eastern parts and lower in the central-south of Hubei province, indicating a profound region-specific change in the incidence rates of MMD (Fig. 3). The county-level AAIR varied largely, ranging from 0 to 25.50 per 100000 person-years. Considering geographic features, we noticed that the high-prevalence counties were mostly distributed in the low hilly areas, nearby the Han River basin and lower reaches of the Yangtze River. In contrast, the low-prevalence counties were mostly located in the Jianghan Plain (Figs. 3 and 1B).

\subsection{Hot-spot Analysis}


The hot-spot analysis revealed both clusters of hot and cold spots of MMD in Hubei province. The hot spots were mainly located in Xiangyang, Wuhan, Huanggang, and Xiaogan City, whereas the cold spots were mainly located in Yichang and Jingzhou City (Fig. 3 and Fig. 1A). Both spots remained minimally changed over time (2017 to 2019), which suggested an environmental effect bias between the spots. As a result, we identified 25 and 17 counties as hot and cold spots, respectively. Surprisingly, the median annual AAIR of hot spots was about 8 times than the cold spots (Table 2), suggesting people in hot spots might be affected by a higher dose of environmental factor. 
Table 2

Comparison of the AAIR and the variables between hot and cold spots.

\begin{tabular}{|c|c|c|c|c|}
\hline Variables & Total $(n=42)$ & $\begin{array}{l}\text { Cold spots }(n= \\
17)\end{array}$ & $\begin{array}{l}\text { Hold spots }(n= \\
25)\end{array}$ & $\begin{array}{l}P \\
\text { value }\end{array}$ \\
\hline \multicolumn{5}{|l|}{$\begin{array}{l}\text { AAIR (per } 100000 \text { person- } \\
\text { years) }\end{array}$} \\
\hline 2017 & $3.7(0.5-5.5)$ & $0.8(0.3-0.9)$ & $5.6(2.9-6.8)$ & $<.001$ \\
\hline 2018 & $4.5(0.9-7.0)$ & $0.9(0.1-1.2)$ & $6.9(3.8-8.7)$ & $\begin{array}{l}< \\
0.001\end{array}$ \\
\hline 2019 & $5.2(0.6-8.3)$ & $1.0(0.3-1.4)$ & $8.1(3.7-12.2)$ & $\stackrel{<}{0.001}$ \\
\hline \multicolumn{5}{|l|}{ Socioeconomic status } \\
\hline Urban population (\%) & $50.2(41.0-59.2)$ & $\begin{array}{l}54.0(48.7- \\
58.7)\end{array}$ & $47.4(33.9-60.5)$ & 0.378 \\
\hline $\begin{array}{l}\text { Per capita DIOUR (1000 \$, } \\
2018)\end{array}$ & $4.7(4.4-5.3)$ & $4.8(4.3-5.4)$ & $4.6(4.5-5.3)$ & 1.000 \\
\hline $\begin{array}{l}\text { Per capita DIORR (1000\$, } \\
\text { 2018) }\end{array}$ & $2.5(2.3-2.9)$ & $2.5(2.0-3.0)$ & $2.5(2.3-2.9)$ & 0.833 \\
\hline Per capita GDP $(1000 \$, 2018)$ & $9.2(4.9-11.5)$ & $10.1(5.5-16.0)$ & $8.6(4.5-11.4)$ & 0.578 \\
\hline $\begin{array}{l}\text { Hospital beds (per } 1000 \\
\text { persons) }\end{array}$ & $5.5(4.5-6.5)$ & $4.9(3.7-6.2)$ & $5.8(4.8-6.5)$ & 0.336 \\
\hline $\begin{array}{l}\text { Medical workers (per 1000- } \\
\text { persons) }\end{array}$ & $5.4(3.9-5.9)$ & $6.0(4.0-8.1)$ & $5.2(3.8-5.6)$ & 0.396 \\
\hline \multicolumn{5}{|l|}{$\begin{array}{l}\text { Particular natural } \\
\text { environments }\end{array}$} \\
\hline Average elevation (m) & $\begin{array}{l}85.1(28.2- \\
152.0)\end{array}$ & $\begin{array}{l}157.4(67.9- \\
533.1)\end{array}$ & $\begin{array}{l}33.6(24.3- \\
104.4)\end{array}$ & 0.001 \\
\hline Rice field (\%) & $49.8(23.9-77.9)$ & $\begin{array}{l}35.7(12.1- \\
56.6)\end{array}$ & $80.3(54.0-99.6)$ & 0.007 \\
\hline Pig density (capita per $\mathrm{km}^{2}$ ) & $\begin{array}{l}202.7(115.9- \\
222.4)\end{array}$ & $\begin{array}{l}188.4(89.3- \\
258.4)\end{array}$ & $\begin{array}{l}218.1(133.7- \\
218.4)\end{array}$ & 0.586 \\
\hline Cattle density (capita per $\mathrm{km}^{2}$ ) & $17.9(2.3-32.0)$ & $7.7(1.6-12.9)$ & $28.9(8.1-48.3)$ & 0.002 \\
\hline \multicolumn{5}{|c|}{$\begin{array}{l}\text { Note: Data are shown as median (IQR) and compared by Wilcoxon rank-sum test. AAIR, age-adjusted } \\
\text { incidence rate. DIOUR, disposable income of urban residents. DIORR, disposable income of rural } \\
\text { residents. GDP, gross domestic product. }\end{array}$} \\
\hline
\end{tabular}

\subsection{Differences Between Hot And Cold Spots}


The potential risk factors compared between the hot and cold spots are listed in Table 2. There was no statistical bias of socioeconomic status or sanitary conditions between the spots. Comparatively, hot spots had lower elevation (33.6 vs $157.4, P=0.001$ ), larger cattle density ( 28.9 vs $7.7, P=0.002$ ), and higher percentages rice field ( $80.3 \%$ vs $35.7 \%, P=0.002)$, indicating that the individuals who live in low elevation regions with close contact to cattle and agricultural water may have a higher risk of MMD.

\section{Discussion}

This study investigated the epidemiological characteristics of MMD in Hubei province, and the first time we applied spatial epidemiology methods in detecting the environmental factors of the disease. Due to lack of the national registry database like Japan and South Korea ${ }^{13,14}$, the incidence of MMD in mainland China has not been clarified. The HHC who collected the inpatient data from all over Hubei, hence we were able to describe a relatively accurate incidence of MMD in this province. Further, Hubei is located in the central China, where the epidemiological characteristics MMD could roughly reflect the prevalence of the disease in mainland China. An early multicenter study in Nanjing, China revealed an average incidence of 0.43 per 100000 person-years from 2000 to $2007^{13}$. In this population-based study, we identified an average annual AAIR of 2.85 per 100000 person-years from 2017 to 2019. MMD was initially described in Japan where the incidence was 0.3 in 1994, 0.54 in 2003 and 0.94 in 2008 per 100 000 person-years ${ }^{15,16}$. In Korea, the incidence was 1.7 to 2.3 per 100000 person-years from 2007 to $2011^{14}$. Moreover, the disease was quite rare in the European and American countries ${ }^{17,18}$.

The genetic factor (especially RNF213) was thought to be the major reason for the geographic distribution difference of $\mathrm{MMD}^{2}$, while our study shed light on the environmental factors that probably participate in the disease. Even if the RNF213 mutation has been detected in 70-90\% of Japanese MMD, it cannot explain the large gap between the prevalence of $R N F 213$ mutation in normal population (1-3\%) and low prevalence of MMD (10 in 100000$)^{4,6}$. In this study, we identified evident regional distribution bias of MMD in Hubei province that the incidence in hot spots is about 8 times than the cold spots. Due to the Chinese Han accounts for the majority (96\%) population in the province, who are supposed to have a similar genetic background. Hence, we concluded that it is the environmental factor that dominantly resulted in the varied geographic distribution of MMD.

Previous studies have demonstrated that leptospirosis could be a risk factor for future cerebrovascular accidents, including $\mathrm{MMD}^{7,8,19,20}$. In 1975, Chinese neurologists Liu et al. from Wuhan Medical College (now is Tongji Medical College of Huazhong University of Science and Technology, Hubei, China), reported 75 patients who underwent cerebral angiography with typical moyamoya features for leptospiral infection, and the author named this disease as LCV ${ }^{7}$. Meanwhile, studies in other provinces (Henan, Anhui, Shandong et al.) where leptospirosis were severely epidemic, also reported a series of cases with LCV (articles in Chinese). Interestingly, the clinical manifestations, pathology, and angiographic findings of LCV were quite similar with MMD mentioned by Japanese neurosurgeons Suzuki et al. in $1969^{7}$. 
Therefore, leptospiral infection was ever recognized as one of the potential causes of MMD, though not has been clarified.

In this study, we focused on whether the hot spots were more suitable for leptospirosis spread under the perspective of ecology. The hot spots mainly located in low mountainous and hilly landform, belonging to the basin of Han River and low reaches of Yangtze River. More importantly, we demonstrated hot spots had lower elevation, larger cattle density, and higher percentages of rice field. The above factors are supposed to increase the risk of leptospirosis outbreak. First, due to the low mountainous and hilly landform, hot spots were supposed to have a larger number of wild or domestic animal hosts for leptospirosis, which would be likely to pollute the water resources ${ }^{9}$. Second, it is acknowledged that the young farmers working manually in the rice field was one of the most important reasons for leptospirosis spread in China ${ }^{10}$. Third, the rich water resources combined with the lower elevation resulted in the individuals in hot spots might have a higher risk of flood disasters, which was a determinant factor for large leptospirosis outbreak ${ }^{10}$. Taken together, we think that individuals who lived in hot spots had a higher risk for leptospirosis outbreak, which might account for why there existed a huge difference of incidence between hot and cold spots.

Actually, the following two observations further point to the association between leptospirosis and MMD. One is that MMD and leptospirosis shared a similar geographic distribution in China. A recent nationwide study revealed that East and Southcentral China had an obviously higher prevalence of MMD than other regions (especially North and Northwest China) ${ }^{21}$, although the authors did not analyze the reasons. Actually, the East and Southcentral China belongs to the Yangtze-Huai River basin and border on the Pacific Ocean, where leptospirosis was high prevalent in the 20th century due to frequent flood disasters and typhoons. On the contrary, the North and Northwest China had relatively less leptospirosis for the dry climate $^{11}$. Thus, it might be the leptospirosis that caused the geographical disparity of MMD. The other is that we identified about $99 \%$ patients with MMD in our observation time from 2017 to 2019 were adults (>18 years old), which means the majority patients were born in the 20th century. Interestingly, the corresponding fact is that the leptospirosis was mainly epidemic in the 20th century and sharply decreased in the 21 st century $^{11}$, which may explain the varied incidence of MMD between adults and children. Therefore, we should attach more importance to the early-life exposure to leptospirosis and MMD.

This study has several limitations. First, the study is based on the ecological analysis, where the association between leptospirosis and MMD is speculative. Second, we did not consider the impact of process of urbanization or population migration, which might result in overestimating the incidence of MMD in the urban regions. However, we noticed that the MMD is more prevalent in the rural areas even this bias might exist, which is in accordance with the fact that the countryside is more affected by leptospirosis. Third, the socioeconomic and environmental variables we collected might change by time, hence there might exist a bias if we use them to reflect the risk of leptospirosis in the early time. For example, the old habit of widely free-ranged pig has been replaced by modern piggery in China, which 
might partly explain the result that we did not find a pig density between hot and cold spots. Thus, we selected the relevant variables (like elevation and rice field) which minimally changed by time so as to reduce this bias as far as possible.

\section{Summary}

This study is the first time in mainland China that we describe the epidemiology of MMD by using the registry database over the province, which would reflect a more accurate incidence of the disease in China. We identified the obvious geographic distribution of MMD in the province, which initially strengthened the importance of environmental factors of this disease. Moreover, we preliminarily identified that people who lived in the low elevation regions with close contact to the cattle and rice field has a high risk of MMD. Future studies are needed to explore the potential environmental factors in MMD, especially for early-life exposure to leptospirosis.

\section{Non-standard Abbreviations And Acronyms}

Moyamoya disease: MMD

Age adjusted incidence rate: AAIR

Ring finger protein 213: RNF213

Hubei Health Committee: HHC

Digital elevation model: DEM

Gross domestic product: GDP

Disposable income of urban residents: DIOUR

Disposable income of rural residents: DIORR

Interquartile range: IQR

leptospiral cerebral vasculitis: LCV

\section{Declarations}

\section{Ethics Statement}

All methods were carried out by following relevant guidelines, and the ethics committee at Zhongnan Hospital of Wuhan University approved this study. Informed consent from all participants was waived due to the study's retrospective nature by the ethics committee at Zhongnan Hospital of Wuhan 
University. All individual identifying information (including name, exact address, and telephone) was anonymized and de-identified to protect patient privacy.

\section{Acknowledgments}

We acknowledge support for data collection from the Hubei Health Committee, China. We thank for having the opportunity to learn knowledge of leptospirosis in the Department of Medical Microbiology and Parasitology, Zhejiang University School of Medicine.

\section{Sources of Funding}

This study was supported by grants from Major Program for Technological Innovation of Hubei Province (Grant No. 2018ACA139).

\section{Disclosures}

None

\section{References}

1. Suzuki, J. \& Takaku, A. Cerebrovascular "moyamoya" disease. Disease showing abnormal net-like vessels in base of brain. Archives of Neurology [Internet]. 1969 [cited 2021 Mar 13];20:288-299. Available from: http://www.ncbi.nlm.nih.gov/pubmed/5775283

2. Kuroda, S. \& Houkin, K. Moyamoya disease: current concepts and future perspectives. The Lancet Neurology. 2008

3. Kim, J. S. Moyamoya disease: Epidemiology, clinical features, and diagnosis. Journal of Stroke, 18, 2-11 (2016).

4. Liu, W. et al. Identification of RNF213 as a susceptibility gene for moyamoya disease and its possible role in vascular development.PLOS ONE. 2011

5. Park, Y. S. et al. The role of RNF213 4810G > a and 4950G > A variants in patients with moyamoya disease in Korea.International Journal of Molecular Sciences. 2017

6. Liu, W., Hitomi, T., Kobayashi, H., Harada, K. H. \& Koizumi, A. Distribution of Moyamoya Disease Susceptibility Polymorphism p.R4810K in RNF213 in East and Southeast Asian Populations.Neurologia Medico-Chirurgica. 2012

7. Liu, X. M. et al. Moyamoya disease caused by leptospiral cerebral arteritis. Chin. Med. J, 93, 599-604 (1980).

8. Matsushima, Y., Qian, L. \& Aoyagi, M. Comparison of Moyamoya disease in Japan and Moyamoya disease (or syndrome) in the People's Republic of China. In: Clinical Neurology and Neurosurgery. 1997.

9. Haake, D. A. \& Levett, P. N. Leptospirosis in humans. Current Topics in Microbiology and Immunology. 2015 
10. Wei, X. Leptospira and Leptospirosis. first ed. The People's Healthy Publishing House, Beijing 1982. (in Chinese)

11. Zhang, C., Wang, H. \& Yan, J. Leptospirosis prevalence in Chinese populations in the last two decades.Microbes and Infection. 2012

12. Morgenstern, H. Ecologic studies in epidemiology: Concepts, principles, and methods. Annual Review of Public Health, 16, 61-81 (1995).

13. Sato, Y., Kazumata, K., Nakatani, E., Houkin, K. \& Kanatani, Y. Characteristics of Moyamoya Disease Based on National Registry Data in Japan., 50, 1973-1980 (2019).

14. Ahn, I. M. et al. Incidence, prevalence, and survival of moyamoya disease in Korea: A nationwide, population-based study., 45, 1090-1095 (2014).

15. Wakai, K. et al. Epidemiological features of Moyamoya disease in Japan: Findings from a nationwide survey. Clinical Neurology and Neurosurgery, 99, 1-5 (1997).

16. Kuriyama, S. et al. Prevalence and clinicoepidemiological features of moyamoya disease in Japan: Findings from a nationwide epidemiological survey., 39, 42-47 (2008).

17. Birkeland, P. et al. Moyamoya disease in a European setting: a Danish population-based study. European Journal of Neurology. 2020

18. Numaguchi, Y. et al. Moyamoya disease in the United States. In: Clinical Neurology and Neurosurgery. 1997.

19. Lessa, I. \& Cortes, E. Cerebrovascular accident as a complication of leptospirosis. The Lancet, 318, 1113 (1981).

20. Li, K-Y. et al. Newly Diagnosed Leptospirosis and Subsequent Hemorrhagic Stroke: A Nationwide Population-Based Cohort Study. Stroke [Internet]. 2021 [cited 2021 Mar 13];52:913-921. Available from: http://www.ncbi.nlm.nih.gov/pubmed/33494640

21. Sun, Y. et al. Incidence and prevalence of moyamoya disease in urban China: A nationwide retrospective cohort study.Stroke and Vascular Neurology. 2021

\section{Figures}



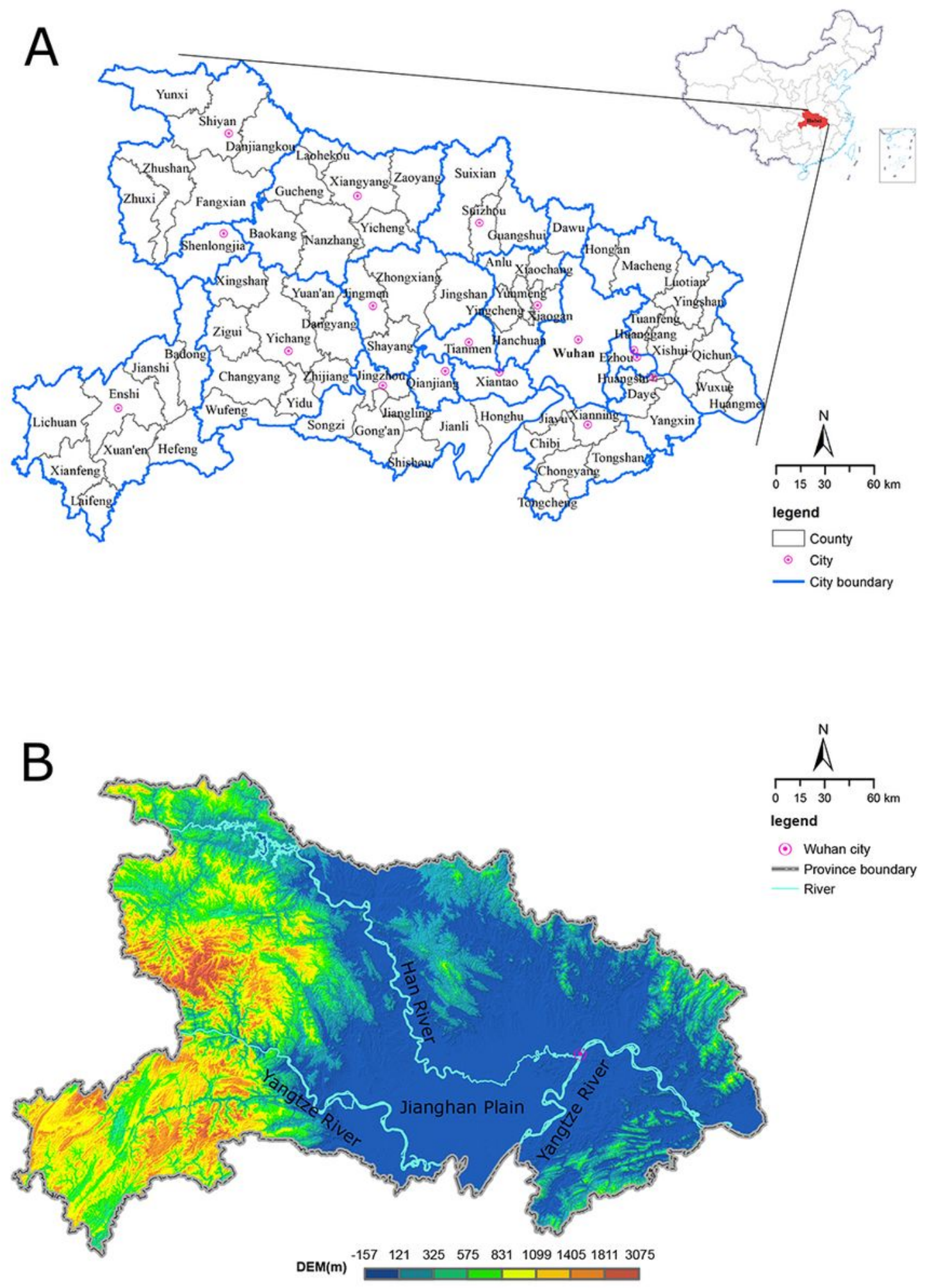

\section{Figure 1}

Map of the study area. A. The administrative division of Hubei province at both county and city level. B. The geographic feature of the province. DEM (digital elevation model), which represents the diverse landforms: hills in the east, mountains in the west, and plain in the south-central. 


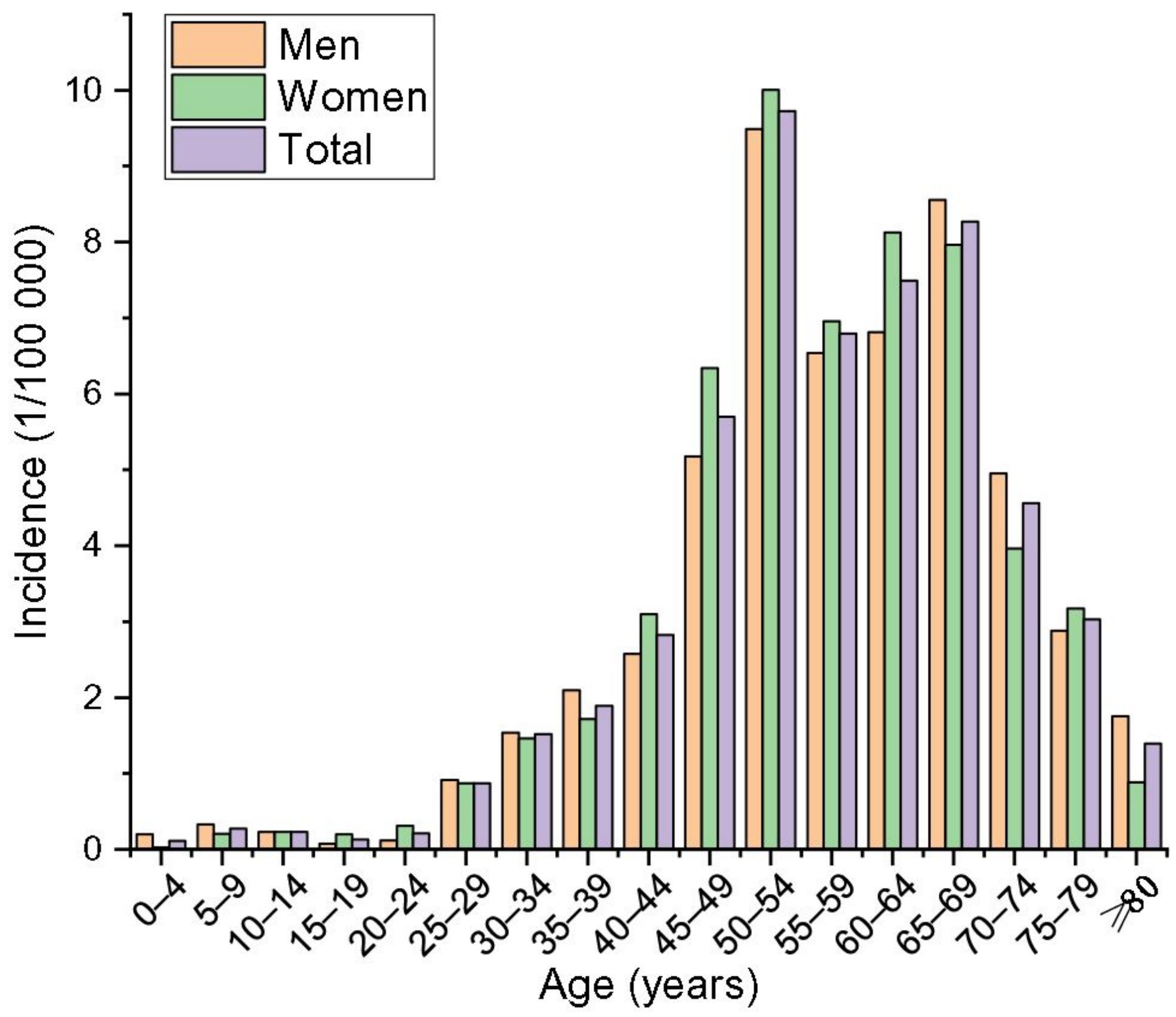

Figure 2

Average annual incidence of MMD by sex and age from 2017 to 2019. "Total" represents sex adjusted incidence in different age groups. 

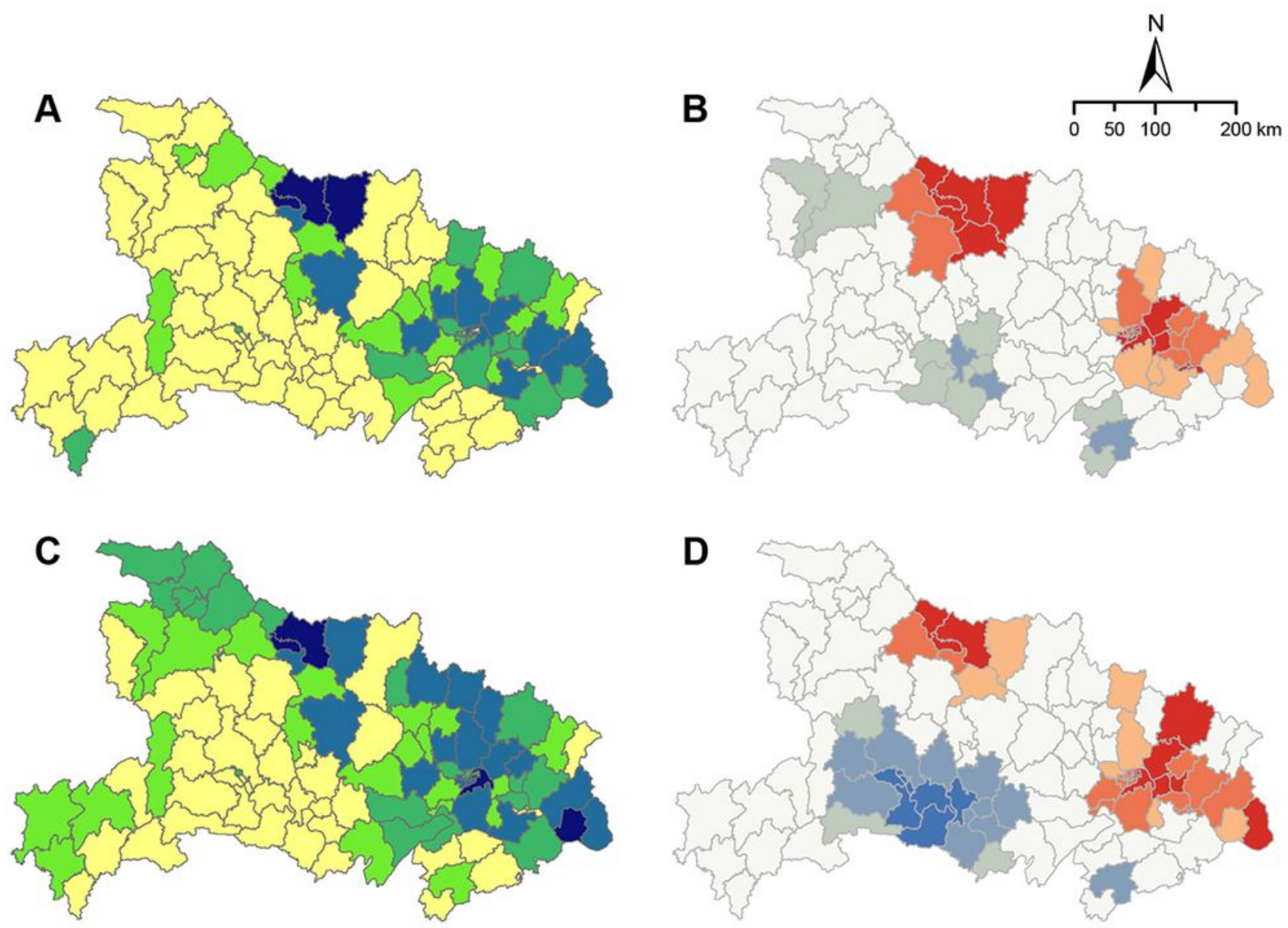

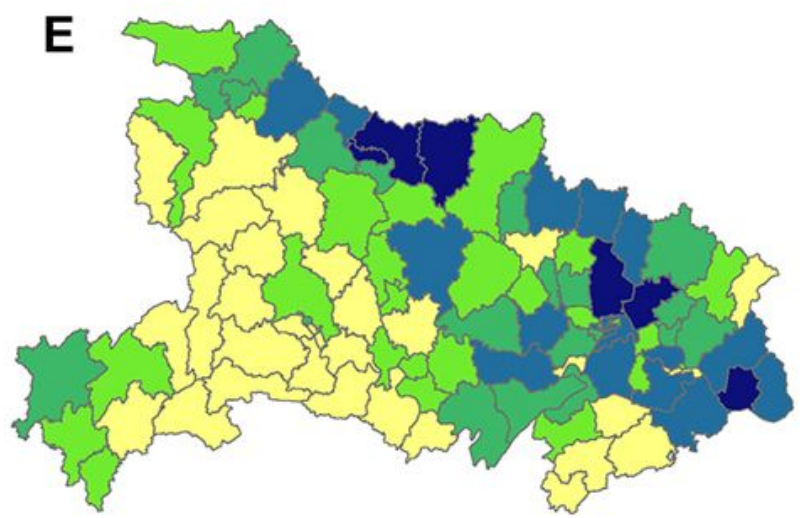

AAIR at county level (per 100000 person-years)

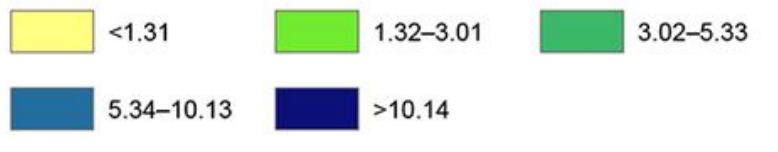

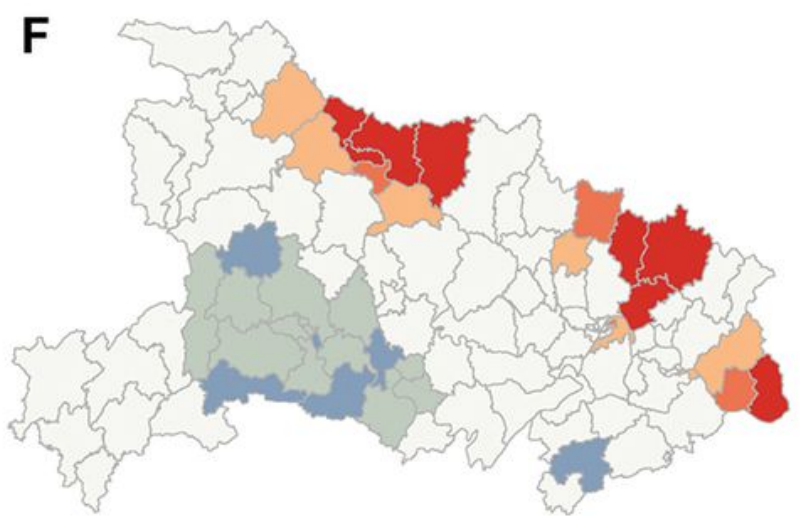

Hot-spot analysis

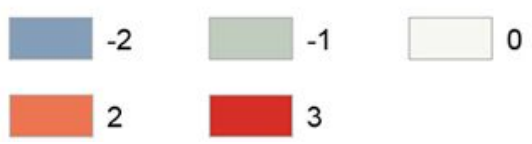

\section{Figure 3}

Age-adjusted incidence rate (AAIR) and hot-spot analysis mapped by county in 2017 (A and B), 2018 (C and D), and 2019 (E and F). The AAIR at the county-level was divided into 5 groups and displayed in different colors, deeper color represents higher MMD incidence. The result of hot spot analysis includes 7 groups from -3 to 3 . The value of $-3,-2$, and -1 represents $99 \%, 95 \%$, and $90 \%$ confidence of cold spot, 
respectively; The value of 3,2, and 1 represents $99 \%, 95 \%$, and $90 \%$ confidence of hot spot, respectively; The value of 0 represents not cluster. 